\title{
High Systemic Levels of the Cytokine-Inducing HMGB1 Isoform Secreted in Severe Macrophage Activation Syndrome
} Karin Palmblad,,$^{*}$ Hanna Schierbeck, ${ }^{1^{*}}$ Erik Sundberg, ${ }^{1}$ Anna-Carin Horne, ${ }^{1}$ Helena Er,
Jan-Inge Henter, ${ }^{3}$ Daniel J Antoine, ${ }^{4}$ and Ulf Andersson ${ }^{1}$
${ }^{1}$ Unit of Pediatric Rheumatology, Department of Women's and Children's Health, ${ }^{2}$ Rheumatol ty L De artment of Medicine, ${ }^{3}$ Childhood Cancer Research Unit, Department of Women's and Children's Health, Karolincka itutet, Karolinska University Hospital, Solna, Karolinska Institutet, Stockholm, Sweden; and ${ }^{4}$ Medical Research Counc Centre Drug Safety Science, Department of Molecular and Clinical Pharmacology, University of Liverpool, United Sin

(MAS) is a potentially fatal complication a n activammation. High mobility group box 1 $(\mathrm{HMGB})$ is a nuclear protein extensively leaked extracellularly during necrotic cell ath or actively secreted by natural killer (NK) cells, macrophages and additional cells during infection or sterile injury. tracellulur HMGB1 orchestrates key events in inflammation as a prototypic alarmin. The redox states of its three cysteines ren der m molecule mutually exclusive functions: fully reduced "all-thiol HMGB1" exerts chemotactic activity; "disulfide HMGB1" has (ytokine-inducing, toll-like receptor 4 (TLR4)-mediated effects-while terminally oxidized "sulfonyl HMGB1" lacks inflammatory activi $y$. This study examines the kinetic pattern of systemic HMGB1 isoform expression during therapy in four children with seve MAS. Three of the four patients with underlying systemic rheumatic diseases were treated with biologics and two suffere rom ingering herpes virus infections at the onset of MAS. All patients required intensive care unit therapy due to life-tr ater. illn ess. Tandem mass-spectrometric analysis revealed dramatically increased systemic levels of the cytokine-ind cing $\mathrm{HMG}_{L}$, isoform during early MAS. Disease control coincided with supplementary etoposide therapy initiated to bonst c. ototic cell death, when systemic HMGB1 levels drastically declined and the molecule emerged mainly in its oxidized, no ninflam, ny isoform. Systemic interferon (IFN)- $\gamma$ and ferritin peaked concomitantly with HMGB1, whereas interleukin (IL)-18 a. monocyte chemotactic protein (MCP)-1 levels developed differently. In conclusion, this work provides new insights in $\mathrm{HM}^{\mathrm{M}} \mathrm{GB} 1$ blu $y$ suggesting that the molecule is not merely a biomarker of inflammation, but most likely also contributes to the pr thogenesis o, MAS. These observations encourage further studies of disulfide HMGB1 antagonists to improve outcome of MAS.

Online address: http://www.molmed.or

doi: $10.2119 /$ molmed.2014.00183

\section{INTRODUCTION}

Macrophage activation s $\mathrm{drc}$ no (MAS) is a severe and $p$ ten $\mathrm{v}$ litethreatening complica $n$ of syst atic inflammatory disord rs. 1. ay occur in response to an infection (ofte, viral), malignancy $f$ rl umatic disease (1).

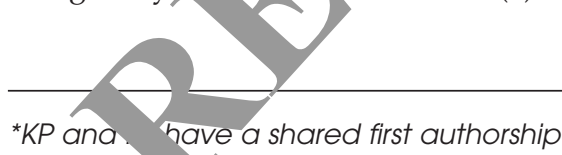

Address co. spondence to Karin Palmblad, Unit of Pediatric Rheumatology, Department of Women's and Children's Health, Karolinska University Hospital, Solna, SE-171 76, Sweden. Phone: (+46) 8 51775613; Fax: (+46) 8 51775562; Email: Karin.Palmblad@ki.se. Submitted September 15, 2014; Accepted for publication September 15, 2014; Epub (www.molmed.org) ahead of print September 16, 2014.

\section{The Feinstein Institute for Medical Research} Empowering Imagination. Pioneering Discovery.
MAS typically appears in patients with systemic onset juvenile idiopathic arthritis (SoJIA) and its adult equivalent, adult-onset Still disease (1); it also is reported in other pediatric inflammatory disorders including juvenile systemic lupus erythematosus (SLE) (2) and 
Table 1. Redox isoforms of HMGB1.

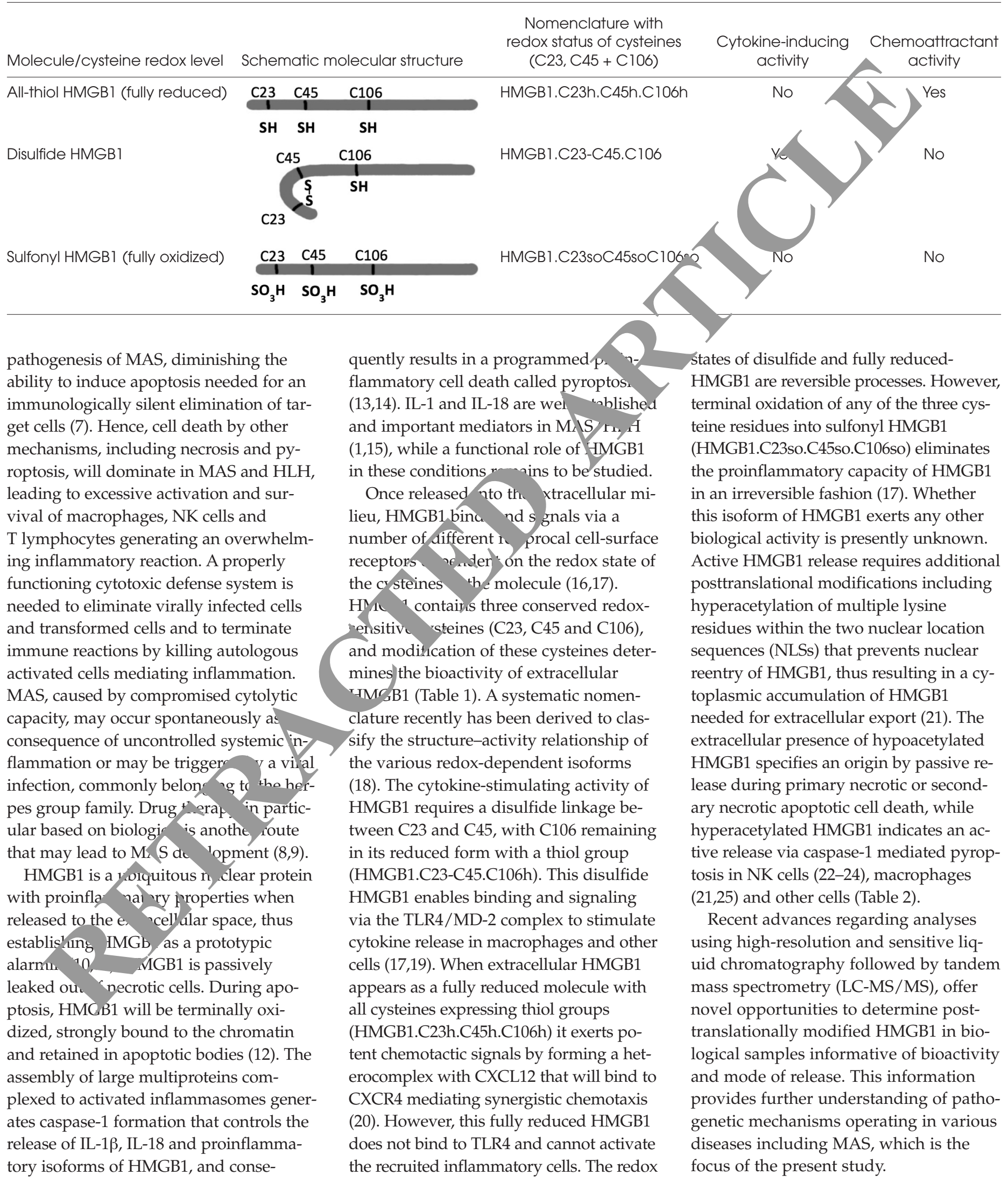


Table 2. Extracellular HMGB1 in clinically relevant modes of cell death.

\begin{tabular}{llll}
\hline \multicolumn{2}{c}{ Apoptosis } & \multicolumn{1}{c}{ Necrosis } & \multicolumn{1}{c}{ Pyroptosis } \\
\hline Immunoreactivity & Noninflammatory & Proinflammatory & Proinflammatory \\
Redox isoform & Sulfonyl HMGB1 & Fully reduced or disulfide HMGB1 & Disulfide HMGB1 \\
NLS sites & Hypoacetylated & Hypoacetylated & Hyperacetylated \\
\hline
\end{tabular}

\section{MATERIALS AND METHODS}

\section{Patients}

Four children aged 3 to 15 years, three previously diagnosed with systemic onset juvenile idiopathic arthritis and one with systemic lupus erythematosus, who all presented with MAS, were studied during a one-year period of November 2010 to October 2011 and fulfilled current MAS criteria $(2,26)$. The diagnosis of SoJIA was made on the basis of the criteria of the International League of Associations for Rheumatology (27) and the SLE patient fulfilled the American College of Rheumatology (ACR) revised SLE criteria. MAS was diagnosed on the combination of clinical features, including cytopenia or sudden decrease in white blood cell counts and/or platelet counts, coagulopathy and liver dysfunction, according to the guidelines proposed by Ravelli et al. and Parodi et al. $(2,26)$. All patients also fulfilled diagnostic criteria for inherited HLH, according to HLH-2004 criteria (28). All patients had markedly elevated inflammatory parameters, including CRP and ferritin, and needed intensive care treatment. Three of the patients had involvement of the central nervous system (CNS), which was severe in two. Pri or , the development of MAS, all four had been administered high doses of ticosteroids, and, in addition, -1osporir. A (CsA), plaquenil, etanercept ing $/$ anakinra. At the time of developing MAS, therapy included tocilizumab and methotrexate; to dizum methotrexate and prednisolone, akin a, CsA and betamethasore; and plà cnil and prednisolone, with oclizu. 1 /lerapy, infections with Epo Barr viy as and varicella-zoster virus $p_{\perp}$ ded the development of MAS.
Owing to the severe clinical MAS presentations including CNS affection in three patients and rapidly progressive pancytopenia in the fourth, an the similarities between MAS and 1 no hagocytic lymphohistiocytosis (HLr 5), w - chose to provide therary with eto ${ }_{\mathrm{H}}$ side and corticosteroids, w is sta dard therapy in HLH $(28,29)$. Ho, ro, since the treatment $\mathrm{p}$ otocols HL $4-94$ and HLH-2004 were o inally lesigned for infants with p. ary 1_._. (familial hemophagocytic rymp. 'istiocytosis, FHL), we administered et poside at lower doses $-120 \mathrm{mg} / \mathrm{m}^{2}$ ) and less frequent intervas (scheduled once weekly) than suggested in the HLH protocols $\left(150 \mathrm{mg} / \mathrm{m}^{2}\right.$, initially twice weekly). The duration of the etoposide treatment ranged from 4 to 7 wks. Although the two patients with severe CNS involvement at onset of etoposide therapy still have impaired CNS function at follow-up, all patients responded with dramatic improvement to the addition of etoposide therapy. The clinical characteristics of the patients are summarized in Table 3.

This study was approved by the Stockholm Ethical Committee, Stockholm,

Table 3. Clinical characteristics.

\begin{tabular}{|c|c|c|c|}
\hline & Patient 2 & Patient 3 & Patient 4 \\
\hline Sex & Male & Female & Female \\
\hline Age at onset of MAS & 5 years & 15 years & 3 years \\
\hline Underlying disease & SOJIA & SLE & SoJIA \\
\hline $\begin{array}{l}\text { Ongoing treatment } \mathrm{g} \\
\text { of MAS }\end{array}$ & $\begin{array}{l}\text { Corticosteroids, } \\
\text { anakinra, CsA }\end{array}$ & Hydroxychloroquine & $\begin{array}{l}\text { Corticosteroids, } \\
\text { tocilizumab, Mtx s.c. }\end{array}$ \\
\hline $\begin{array}{l}\text { Verified infect } \\
\text { onset }\end{array}$ & None & None & VZV \\
\hline Diagnostic criten & Yes & Yes & Yes \\
\hline Diagno stic o teria $\mathrm{H}_{2} \_\mathrm{H}$ & Yes & Yes & Yes \\
\hline Neurolo. al sym.,pton & No & Severe & Severe \\
\hline First-line Mr 'Kerapy & MP-pulses & MP-pulses & MP-pulses \\
\hline Etoposide & $\begin{array}{l}50-100 \mathrm{mg} / \mathrm{m}^{2} / \text { dose for } \\
6 \mathrm{wks}\end{array}$ & $\begin{array}{l}50-75 \mathrm{mg} / \mathrm{m}^{2} / \text { dose for } \\
5 \mathrm{wks}\end{array}$ & $\begin{array}{l}70 \mathrm{mg} / \mathrm{m}^{2} / \text { dose for } \\
4 \mathrm{wks}\end{array}$ \\
\hline Additional treatment & $\begin{array}{l}\text { ICU care, } \\
\text { corticosteroids }\end{array}$ & $\begin{array}{l}\text { ICU care, corticosteroids, } \\
\text { CsA }\end{array}$ & $\begin{array}{l}\text { ICU care, } \\
\text { corticosteroids }\end{array}$ \\
\hline Clinical response & Complete & Severe CNS sequele & Partial CNS sequele \\
\hline
\end{tabular}

Mtx, methotrexate; s.c., subcutaneously; EBV, Epstein-Barr virus; VZV, varicella-zoster virus; MP-pulses, methylprednisolone pulses; ICU, intensive care unit. 
Sweden. Parents and patients gave informed consent before inclusion.

\section{Blood Samples}

Sera were obtained from blood samples collected in tubes without additive and plasma was collected in EDTA tubes. Blood samples were centrifuged at $1440 \mathrm{~g}$ for $10 \mathrm{~min}$ and cells were removed and stored at $-80^{\circ} \mathrm{C}$ until assayed.

We serially determined the serum levels of inflammasome-associated HMGB1, IL- $1 \alpha$, IL-1 $\beta$ and IL-18 and additionally IFN- $\gamma, \mathrm{MCP}-1$ and ferritin in the patients before, during and after therapeutic intervention until inflammation had resolved.

Serum samples from patients with oligoarticular, polyarticular, enthesitisrelated or other type of JIA according to the ILAR criteria, as well as serum samples from 10 healthy pediatric controls (range 2-14 years), were collected at Astrid Lindgren Children's Hospital and analyzed for comparison of total HMGB1 levels.

\section{ELISA Assays for HMGB 1 Detection}

HMGB1 levels were measured in undiluted plasma using HMGB1 enzymelinked immunosorbent assay (ELISA) kit II, according to the instructions of the manufacturer (IBL International, Hamburg, Germany). The lower limit for tection was $0.3 \mathrm{ng} / \mathrm{mL}$.

\section{Cytometric Bead Array ( (A) and Bioplex for Detection :IFN IL-I $\alpha$, IL- $1 \beta$, IL-18 and MCr}

All sera were dilated $1: 8$ before

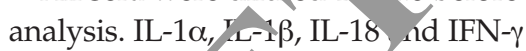
were measur by Bioplex (Bio-Rad Laboratories, Hercult $\mathrm{SA}$, JSA) with a lower detection li t of $4-\mathrm{pg} / \mathrm{mL}$. Levels of $\mathrm{MCP}=1$ or ured by CBA using Human So ble Protein Flex Sets and Human Solvole Protein Master Buffer Kits (BD Biosciences, San Jose, CA, USA) with a lower detection limit of $20 \mathrm{pg} / \mathrm{mL}$. All analyses were performed according to the instructions of the manufacturers.

Serum levels of ferritin were analyzed at the clinical laboratory at Karolinska University Hospital in Stockholm, Sweden.

\section{Characterization of HMGBI Isoforms by Electrospray lonization-Liquid Chromatography-Tandem Mass Spectrometry (ESI-LC-MS/MS)}

All chemicals and solvents used were of the highest available grade (SigmaAldrich, St. Louis, MO, USA). Samples were precleared with $50-\mu \mathrm{L}$ Protein G-Sepharose beads for $1 \mathrm{~h}$ at $4^{\circ} \mathrm{C}$. HMGB1 present in $100 \mu \mathrm{L}$ serum was immunoprecipitated with $5 \mu \mathrm{g}$ of rabbit anti-HMGB1 (ab18256; Abcam, Cambridge, UK) for $16 \mathrm{~h}$ at $4^{\circ} \mathrm{C}$ as described previously (30). For the analysis of HMGB1 redox posttranslational modifications, free thiol groups within $\mathrm{HM}_{\mathrm{A}} \mathrm{G}$ were alkylated with $90 \mathrm{mmol} / \mathrm{L}$ $\mathrm{N}$-ethylmaleimide (NEM) at $4^{\circ} \mathrm{C}$ for $10 \mathrm{~min}$. Cysteine residues in 1 fide bonds were then reduced with $30 / \mathrm{nu}_{\mathrm{r}} \mathrm{ol} / \mathrm{L}$ dithiothreitol (DTT) at $4^{\circ} \mathrm{C}$ for $1 \mathrm{~h}$, Newly DTT reduce steines then were alkylated with a eavy eled $\mathrm{d}_{5} \mathrm{NEM}$ $(\mathrm{NEM}+5 \times$ deute $n) \mathrm{n}$, nich yields a mass shift of 130 ator. mass unit (amu) comparea it he NeM-unexposed protein nd 5 an ompared with the NEMall $y$, d proten. Terminal oxidation of HMGB cteines was characterized as described previously $(16,17,31)$. Samples were ubjected to trypsin (Promega, Madison, WI, USA) or GluC (New England Biolabs, Ipswich, MA, USA) digestion according to manufacturer's instructions and desalted using C18 zip-tips (Millipore, Billerica, MA, USA). Assessment of HMGB1 acetyl modifications was determined as previously described (32). Peptide analysis was determined as described previously using an $\mathrm{AB}$ Sciex QTRAP 5500 equipped with a NanoSpray II source (AB Sciex, Framingham, MA, USA) by inline liquid chromatography using a U3000 HPLC System (Dionex [Thermo Scientific], Sunnyvale, CA, USA), connected to a $180 \mu \mathrm{m} \times 20 \mathrm{~mm}$ nanoAcquity UPLC C $\mathrm{C}_{18}$ trap column and a $75 \mu \mathrm{m} \times 15 \mathrm{~cm}$ nanoAcquity UPLC BEH130 $\mathrm{C}_{18}$ column (Waters, Milford, MA, USA) via reducing unions. A gradient from $0.05 \%$ trifluoroacetic acid (TFA) (v/v) to $50 \%$ acetonitrile $(\mathrm{ACN}) / 0.08 \%$ TFA $(\mathrm{v} / \mathrm{v})$ in $40 \mathrm{~min}$ was applied at a flow rate of $200 \mathrm{~nL} / \mathrm{min}$. The ion spray potential was set to 2,200-3,500 V, the nebulizer gas to 19 and the interface heate to $150^{\circ} \mathrm{C}$.

\section{RESULTS}

Four children with excu conally severe MAS (clinicar ta are d tlined in Table 3) were stu iled retı ctively regarding system c overall H.MGB1 levels and the kinetic pression of systemic HMGB1 isc ms guncrated by posttranslational modi tions in particular. First-line therэpy based on high intravenous doses of tigosteroids and cyclosporine A (CsA) tosuppress the cytokine storm did not control the excessive inflammation in any of the MAS patients. Systemic etoposide treatment subsequently was introduced to improve the deficient ability to mediate apoptosis in critical target cells driving the uncontrolled inflammation. Particular focus was directed on HMGB1 isoform analysis in blood samples obtained closely before and after etoposide infusions. Information about the acetylation state of the extracellular HMGB1 discriminates between active and passive modes of release (Table 2), while HMGB1 redox isoform analysis relates to reciprocal receptor usage and thus the functional role of HMGB1 in the inflammatory process (Table 1). Studies of well-known proinflammatory mediators and biomarkers known to reflect the clinical course of MAS were conducted in parallel. These serum analyses included assessments of ferritin, IFN- $\gamma$, IL-1 $\alpha$, IL-1 $\beta$, IL-18 and monocyte chemotactic protein (MCP-1).

Total serum HMGB1 levels in MAS patients before initiation of etoposide were significantly higher $(p<0.05)$ compared with those observed in JIA patients without MAS and healthy children (Figure 1). Peak systemic HMGB1 levels were recorded at the same time as the MAS patients expressed maximal symptoms and signs. The predominant part of the total HMGB1 pool was at this stage hyperacetylated (Figures 2A, 3A, 4A, 5A) indicating that the systemic HMGB1 in these patients was actively secreted rather than passively released from dying cells $(14,21)$. 


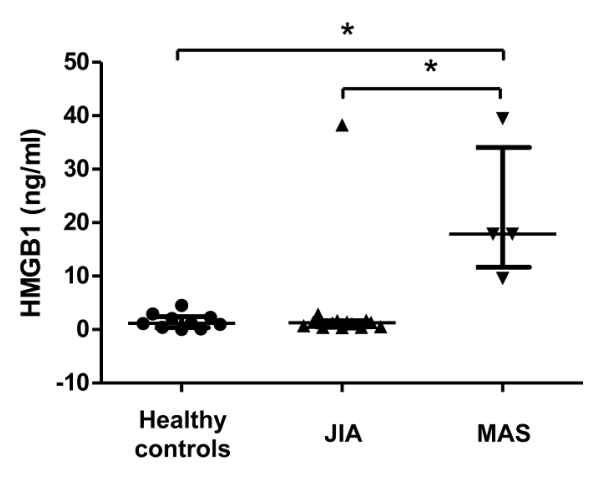

Figure 1. High systemic HMGB1 levels in MAS patients. Serum concentrations of total HMGB1 levels were markedly increased in plasma during severe MAS as compared with children with uncomplicated JIA and healthy pediatric controls. HMGB1 levels were measured by ELISA and correlation was calculated using the Spearman rank correlation test. The HMGB1 levels in the same cohort of JIA patients and healthy control children have been published previously in (38).

Control of inflammation with a clinical stabilization in the patients coincided with the initiation of supplementary etoposide treatment, when a prompt decrease of the systemic HMGB1 levels occurred. Redox state analysis of the expressed HMGB1 isoforms revealed additional useful clinical information. Serum samples taken fore initiation of etoposide treatment mainly expressed the fully red and disulfide HMGB1 isoforms these icoforms mediate inflamma in vromotion of chemotactic sic ls, accur clating professional inflam, ato, ells or activating these via the IDR4 recep or complex, respectively. $\mathrm{Tll}$ de $\mathrm{FMGB1}$ binds to the extracellular apto protein MD-2 of the TLP 4 re ptor a mplex, in the same fashion $1 ;$ saccharide (LPS), generating po proinflammatory cytokine production, which is one of the hallmarks of MAS. By contrast, serum samples obtained after etoposide treatment predominantly expressed HMGB1 that was terminally oxidized expressing sulfonic acid $\left(\mathrm{SO}_{3} \mathrm{H}\right)$ groups on the cysteine residues (Figure 2B, 3B, 4B, 5B). This is an HMGB1 isoform without known proinflammatory
A
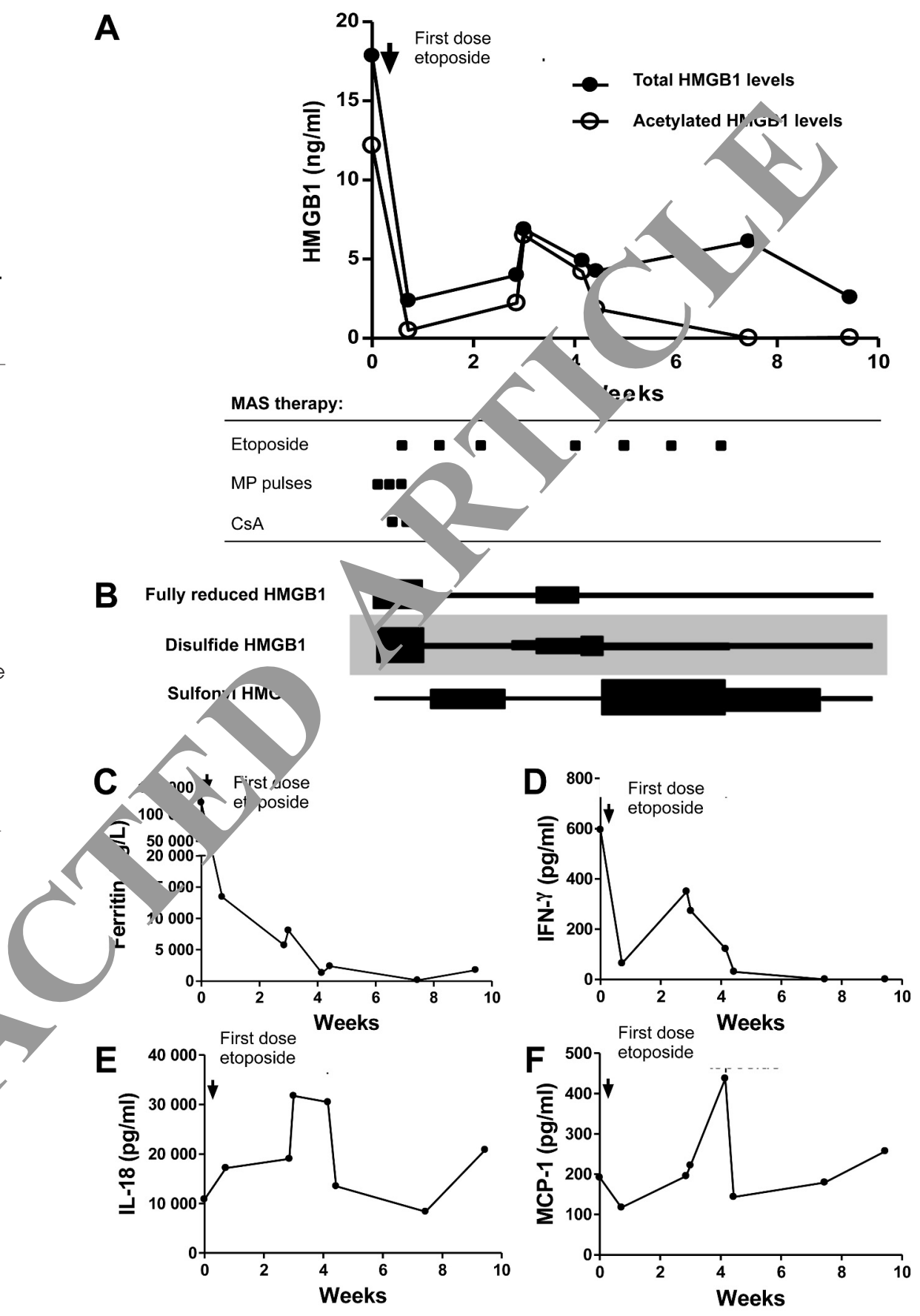

Figure 2. Longitudinal serum analyses before and after introduction of etoposide treatment in patient 1. Serum concentration of total HMGB1 and hyperacetylated HMGB1 during the course of MAS are demonstrated in (A) with the different HMGB1 redox-forms illustrated in (B). High levels of HMGB1 in both fully reduced and disulfide isoforms were documented during severe disease, which rapidly declined after initiation of etoposide treatment concomitantly with serum concentrations of (C) the clinical biomarker ferritin as well as (D) IFN- $\gamma$. In the clinical resolution phase, serum HMGBI appeared predominantly in the terminally oxidized sulfonyl isoform. Serum concentrations of (E) IL-18 and (F) MCP-1 peaked weeks later when the patient was recovering. MP-pulses: methylprednisolone pulses. 
A

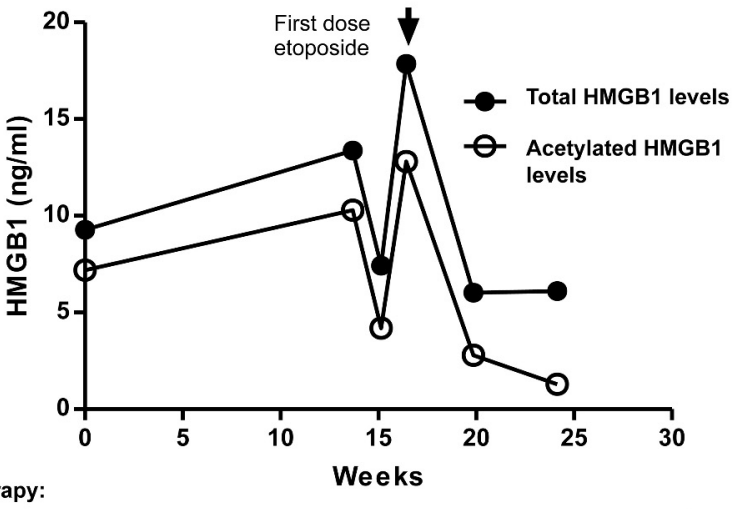

MAS therapy:

-

MP pulses

CsA

B Fully reduced HMGB1

Disulfide HMGB1

Sulfonyl HMGB1
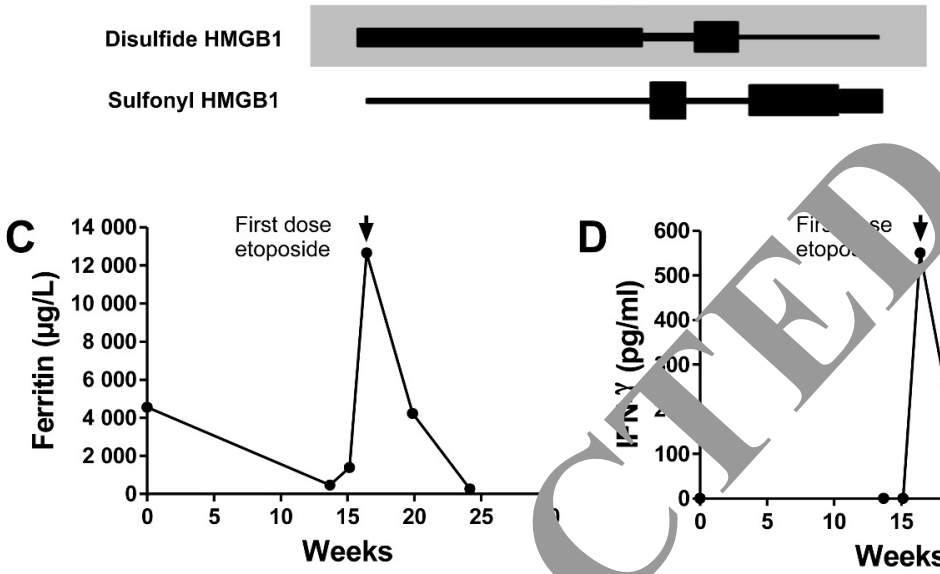

$E$
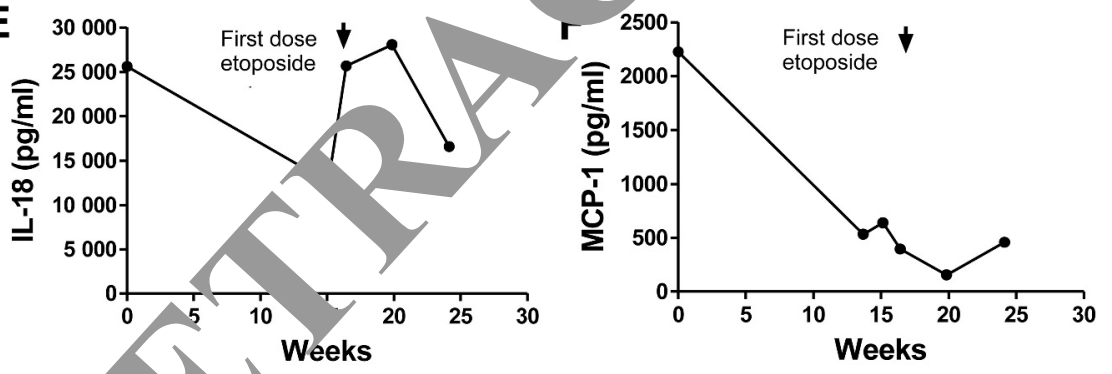

Figurc hal serum analyses before and after introduction of etoposide treatment in $\mathrm{A}$ ent 2. Serum concentration of total HMGB1 and hyperacetylated HMGB1 are illustrate, in ( $A$ ) with the different HMGB1 redox-forms illustrated in (B). The first two serum samples were collected at onset of SoJIA without MAS manifestations when HMGB1 levels appeared mainly in its cytokine-inducing, hyperacetylated isoform. These HMGB1 levels increased at onset of MAS and declined promptly after treatment with etoposide infusions. Increased serum concentrations of (C) ferritin and (D) IFN- $\gamma$ were documented during MAS followed by a normalization post etoposide treatment. (E) Serum IL-18 levels were distinctly increased during the entire observation period with peak values during MAS. (F) MCP-1 did not mimic the clinical course. MP-pulses: methylprednisolone pulses. activities. The clinical signs of disease were considerably milder at this stage. Taken together, these findings suggest that etoposide treatment co mibuted to a reduction of total HMr 81 yels and a conversion of the remaint noninflammatory isoform

Serum ferr is a pi tein that stores iron in a soruble, $\mathrm{m}$ / and its synthesis is regula d by intracellular iron, inflammatory tokin s and oxidative stress. se $n$ fencul levels are especially increas in HLH and MAS, since hemovhagocy tosis results in enhanced uptake haptoglobin-hemoglobin complexes by macrophages triggering a production of ferritin to sequester the excessive amount of free iron. Serum ferritin levels are used as the golden standard parameter to monitor the clinical course of MAS/HLH $(33,34)$. All our studied MAS patients expressed serum ferritin levels that followed a parallel course to that of hyperacetylated serum HMGB1 levels and all these parameters mirrored the clinical course with decreased levels in response to etoposide treatment combined with clinical improvement (Figures 2C, 3C, 4C, 5C). Patient 1 in particular demonstrated a dramatic decline in serum ferritin levels from 121,937 to $13,416 \mu \mathrm{g} / \mathrm{L}$ (upper normal range should be below $100 \mu \mathrm{g} / \mathrm{L}$ ) within a few days after etoposide infusions (see Figure 2C).

IFN- $\gamma$ is the main macrophage-activating cytokine and is released from activated NK cells and T lymphocytes. IFN- $\gamma$ primes the capacity of macrophages for phagocytosis and for proinflammatory cytokine production and is thus a central cytokine in the pathogenesis of MAS $(1,35)$. Serum levels of IFN- $\gamma$ were increased markedly during periods with severe disease in all studied MAS patients and these levels promptly decreased during clinical resolution after etoposide treatment (Figures 2D, 3D, 4D, 5C).

IL-1 and IL-18 also have been demonstrated to be central pathogenic mediators in MAS $(36,37)$ and both molecules are released during pyroptosis, which likewise is an important pathway for HMGB1 release $(13,14)$. Increased serum levels of IL- 
A

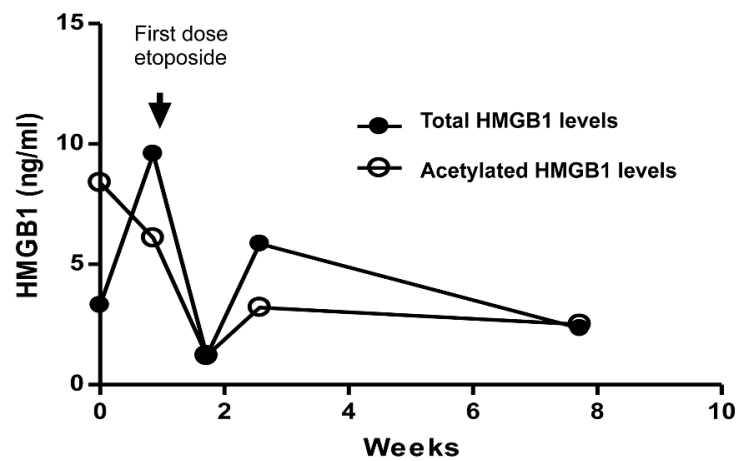

MAS therapy:

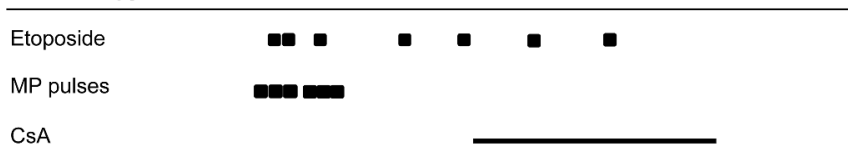

B

CsA

B Fully reduced HMGB1

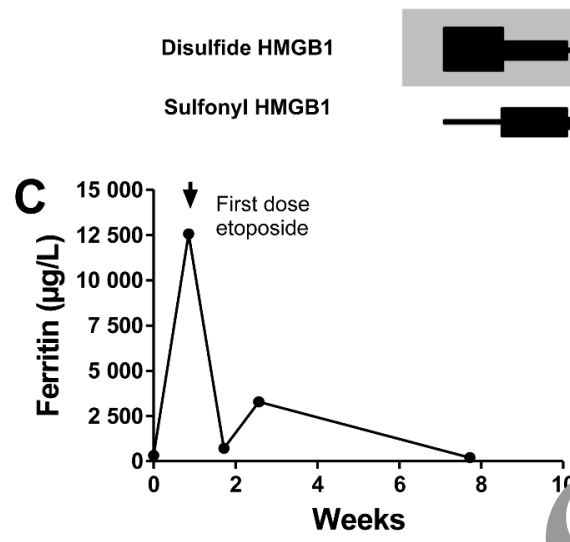

E
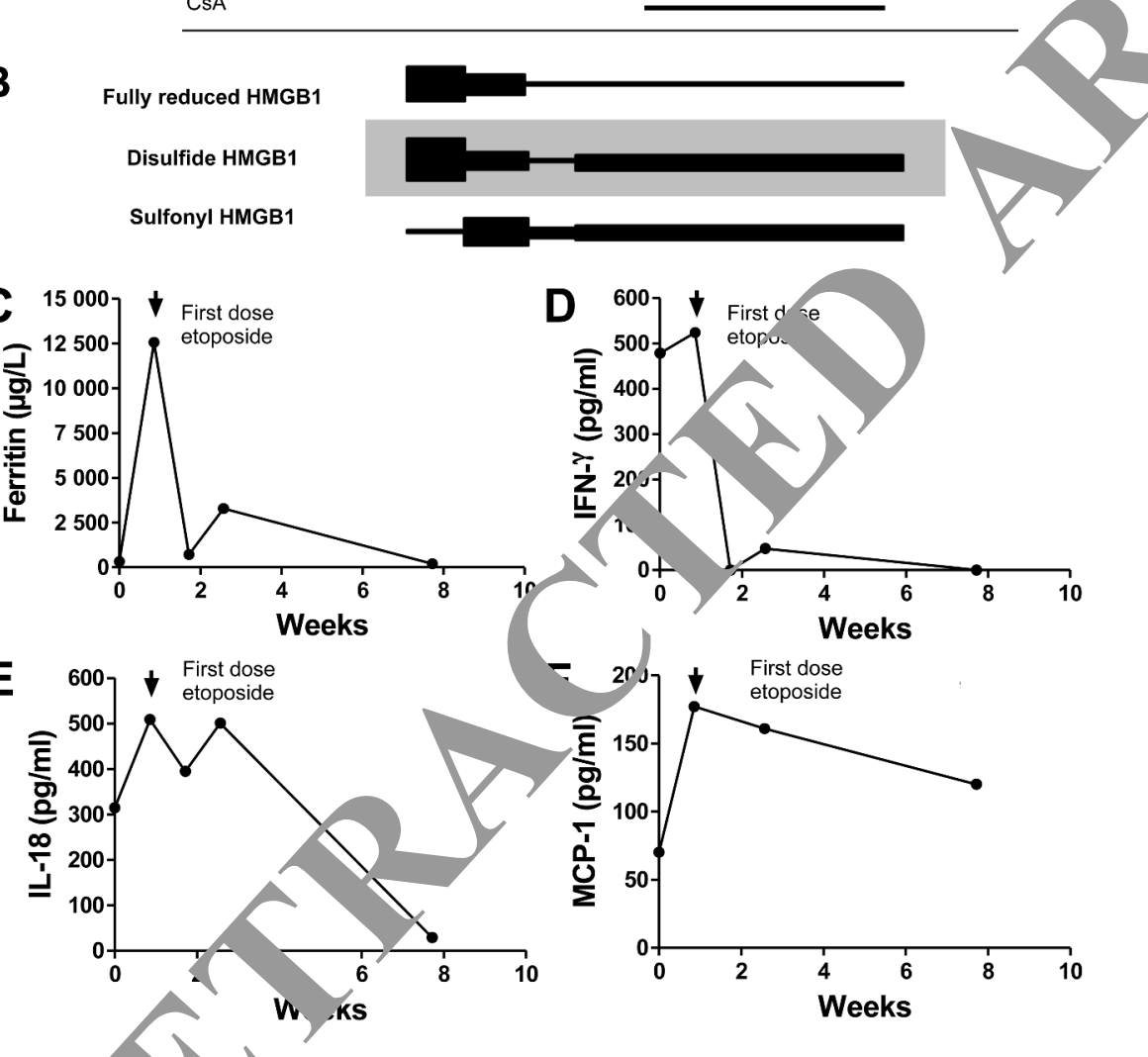

Figure 4. Longit al se um analyses before and after introduction of etoposide treatment in nt 3. am concentration of total $\mathrm{HMGB1}$ and hyperacetylated HMGBI are demc trate 4 in (A) with the different $H M G B 1$ redox-forms illustrated in (B). The expression of serum, els or HMGBI as well as (C) ferritin and (D) IFN- $\gamma$ corresponded very well to the clinical urse of MAS with a rapid decline and clinical improvement after etoposide treatment. IL-18 and MCP-1 levels were increased during critical disease with a prolonged release period (E,F). CsA: cyclosporine A; MP-pulses: methylprednisolone pulses.

18 were documented in all our patients, with peak values appearing later than serum HMGB1, IFN- $\gamma$ and ferritin levels (Figures 2E, 3E, 4E, 5E). Serum IL-1 $\alpha$ and
IL-1 $\beta$ were not detected at any time point in any of the studied MAS patients (data not shown). IL-1 exerts potent biological effects at the low $\mathrm{pg} / \mathrm{mL}$ range, and we believe that our detection methods were not sensitive enough to discover these low levels. The chemokine MCP-1 is increased in JIA patients ( 8 ) and has been implicated in the pa go nesis of HLH where serum concent to disease actiyity (39). In ce present study, serum TP-1 le) als did not change in any consistent ner in response to treatm nt and the levels peaked around $3-4$ wh fter the initiation of therapy (i. res $2,, 1,4 \mathrm{~F}, 5 \mathrm{~F}$ ).

To. nmarize, the results were uniform in all four MAS patients with high 1. ols of inflammatory HMGB1 isoforms promptly declining during clinical resoIution induced by therapeutic intervention with etoposide, corticosteroids and cyclosporin A. Systemic levels of ferritin and IFN- $\gamma$ appeared concomitantly with HMGB1, whereas levels of IL-18 and MCP-1 did not correlate as closely with the clinical course.

\section{DISCUSSION}

In this report, we present four children with severe MAS, where serum levels of total HMGB1 and its redox isoforms were monitored longitudinally at clinically important stages. We revealed markedly elevated serum HMGB1 levels in active MAS in all patients. Moreover, the systemic HMGB1 was expressed in immunological active isoforms promoting either cytokine induction, phagocytosis or chemotaxis during critical illness. Control of clinical inflammation was achieved in all patients when etoposide treatment was added to the conventional antiinflammatory treatment with a marked decline of the extracellular presence of HMGB1 that then mainly appeared irreversibly oxidized to the inflammatory inactive isoform, sulfonyl HMGB1. This in line with several publications that have demonstrated that the oxidative stress during apoptotic cell death induces potent intracellular oxidation events resulting in terminal oxidation of HMGB1 (40-42). Sulfonyl HMGB1 is generally the end product of apoptotic cell death and shows caspase-3 dependence (40). Etoposide is a chemotherapeutic drug that inhibits topoisomerase II, resulting in er- 
A

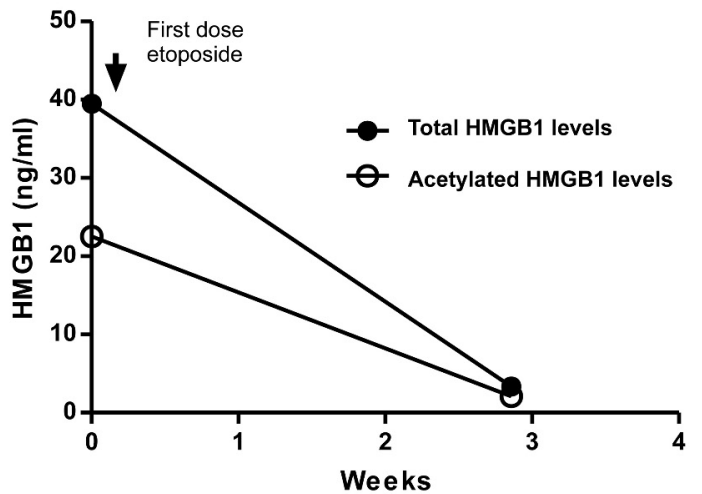

MAS therapy:

\begin{tabular}{llll}
\hline Etoposide & - \\
MP pulses & a
\end{tabular}

B

B Fully reduced HMGB1

\section{Disulfide HMGB1 \\ Sulfonyl HMGB1}
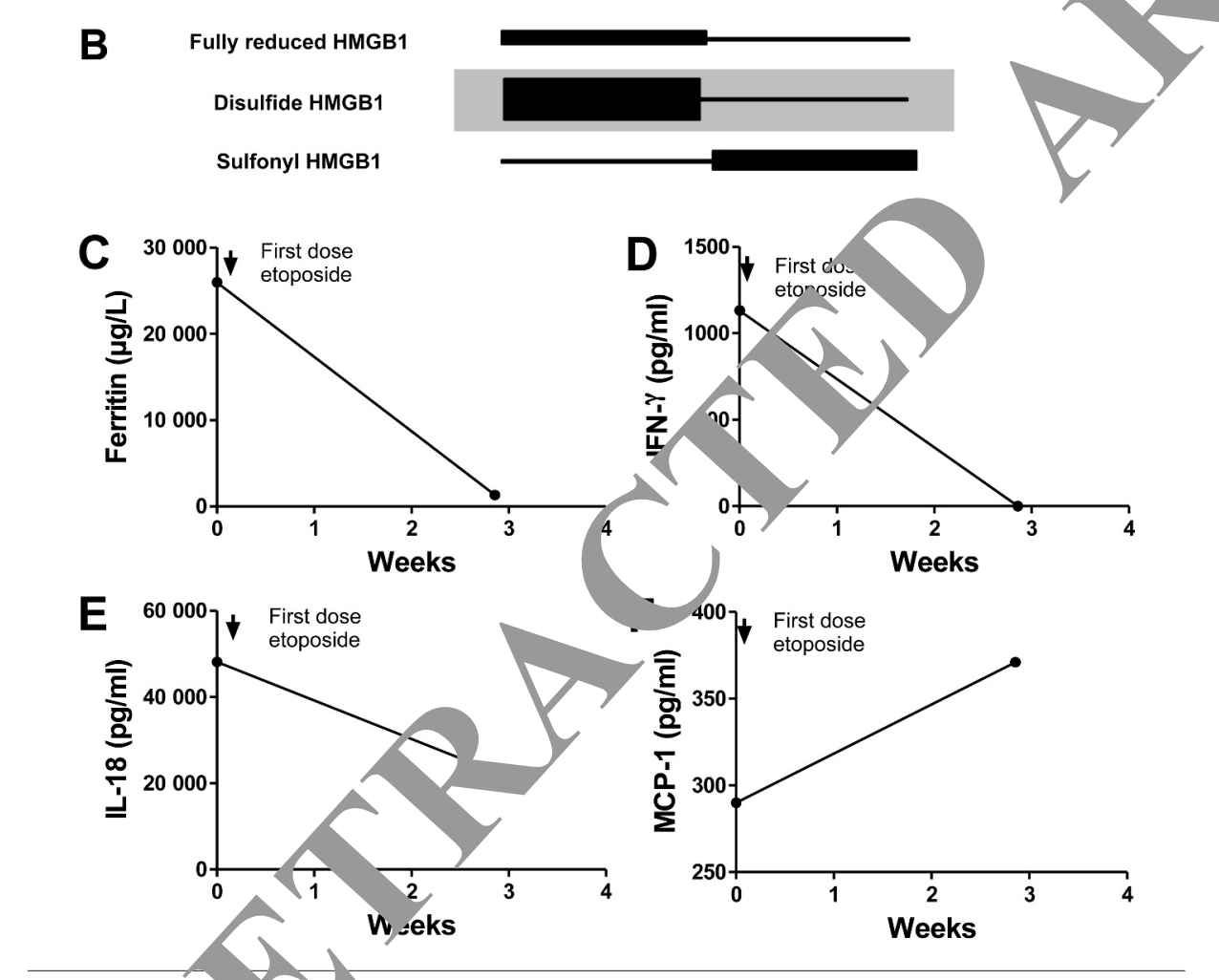

Figure 5. Serum $\mathrm{vses}$ in patient 4 before and after treatment with etoposide. Serum concerira ${ }_{1}$, of to il HMGB1 and hyperacetylated HMGB1 are presented in (A) with the differe. dox-forms illustrated in (B). Two serum samples were analyzed where normalize vvels of (A) HMGBI, (C) ferritin and (D) IFN- $\gamma$ were documented after intervention with etoposide and subsequent clinical improvement. IL-18 declined but was still elevated (E) while MCP-1 increased (F). MP-pulses: methylprednisolone pulses.

rors in DNA synthesis leading to apoptotic cell deaths in rapidly dividing or activated cells. It was recently demonstrated in an animal HLH model based on a lymphocytic choriomeningitis virus infection in perforin-deficient mice that etoposide selectively ablated activated pathogenic $\mathrm{T}$ cells via induction of apoptotic cell death. Resting $\mathrm{T}$ cells, including memory $\mathrm{T}$ cells, were not influenced by the treat- ment (43). Etoposide is the mainstay of treatment for HLH patients (44), but has not yet undergone controlled trials for MAS patients. The life-Areatening courses of MAS in our patie $v$ ho were refractory to high-dose cort additional treacment optic o. Since the clinical expres ns of $\mathrm{F} \mathrm{LH}$ and MAS are indisting, alstrable, chose etoposide in a somen rat reduced dose than commonly used fc HLH treatment. A considerable ns agar. the use of etoposide is an elimu ion of too many immunocompetent cello necessary for the defense against h hogens, as in patient \#2 who developed neatropenia and bacterial sepsis after the etoposide infusion, and was successfully treated. All patients had ongoing immunomodulatory therapy at onset of MAS when additional treatment with intravenous corticosteroid pulses only or corticosteroid pulses plus CsA was initiated. Inadequate clinical responses in the treated patients led to supplementary etoposide treatment that was followed by drastically reduced HMGB1 levels, terminal HMGB1 oxidation and clinical improvement. Whether the observed outcome parameters were due to effect generated by etoposide alone or etoposide acting synergistically with other therapeutic agents cannot be resolved in our small study.

Significant amounts of extracellular HMGB1 may be discharged either by active secretion from activated immune cells or by passive release from necrotic, pyroptotic or damaged cells, but not from apoptotic cells (10). Acetylation of multiple lysine residues within the two NLS sites of HMGB1 is a key regulatory mechanism promoting the active release of HMGB1 from secreting cells. The high levels of hyperacetylated HMGB1 during severe MAS imply that the largest contribution to the systemic HMGB1 pool came via secretion from stimulated professional immune cells. The systemic levels of hyperacetylated HMGB1 demonstrated in our MAS patients were exceptionally high and even exceeded levels recorded when identical LC-MS/MS methods were employed to assess acetylated HMGB1 serum levels in acetaminophen (APAP)-intoxicated pa- 
tients, who either died or needed a liver transplant (45). It is well established that acetylated HMGB1 is a major pathogenetic factor causing liver failure during APAP intoxication $(30,42,45)$. Serum levels of acetylated HMGB1 have been demonstrated to be a highly sensitive and specific biomarker to predict the clinical outcome in patients with APAP-induced hepatotoxicity (45). Future prospective studies are warranted to estimate the value of assessing acetylated HMGB1 levels in

MAS/HLH as a prognostic tool to predict outcome in these conditions.

\section{CONCLUSION}

How could then extracellular HMGB1 contribute to the systemic inflammation in MAS? There are several HMGB1-dependent pathways that may be involved if we postulate that the initiating event in MAS in a given sterile or infectious insult is an impaired capacity to provide key cytolytic molecules needed for induction of apoptosis in critical target cells. Other modes of cell deaths including necrosis or pyroptosis will then take place instead. These cellular events will generate extracellular fully reduced HMGB1 as well as disulfide HMGB1 pools that will drive inflammation via chemotactic signals via CXCR4 (20) and activation of the recruited inflammatory cells via TLR4/MD2 $(19,46)$ to produce proinflum matory cytokines and to indy oweriul phagocytic responses. A de vien ar. tolytic capacity to elimir te a logous activated NK cells an vtotoxic cells, which are key proa icer "1s of both IFN- $\gamma$ and HMC $/ \mathrm{DI}$, will fu, ner contribute to the cr phatge activation in the patients. The "mos identical temporal charges bserve, in our patients regardin aevels of IFN- $\gamma$ and acetylatea MGB1 in response to therapy are in line with this assumption (Figures 2-5). It is important to consider that it is most likely only the cytolytic pathway that is selectively compromised in cytotoxic NK cells and CD8-positive T cells in MAS/HLH patients. When these cells get activated they will produce much IFN- $\gamma$ and HMGB1 and other mediators in their normal repertoire. Furthermore, the poor cytolytic activity seen in HLH/MAS patients may lead to a failure to remove autologous virus-infected cells and thus the source for antigen stimulation will persist leading to long-lasting antigen-driven activation of the immune system escalating the inflammatory response.

"HMGB1 with a disulfide linkage between cysteine 23 and 45 binds to MD-2 of the TLR4 receptor complex with almost the same avidity as LPS" (2015 letter from Huan Yang to Ulf Andersson; unreferenced; see Acknowledgments) (46). Consequently, high systemic disulfide HMGB1 levels may elicit a cytokin. storm analogous to that occurring gram negative sepsis $(46,47)$. Furthermore, HMGB1 has an inherer rility to form immunostimulatory compleses, with other molecules including nucle c acids, nucleosomes, IL-1 $\alpha$ - $1 \beta$, or CXCL12 as well as exogeno facto including LPS and additional TL igan s, thereby enhancing proinflamma y responses in a synergist via eciprocal receptors for the FMG nzrtner molecules $(48,49)$. ro + inflam masome activation during xagger 4 pyroptotic cell death is another mechanism that may contribute with IL-1 $\beta$, IL-18 and proinflammatory HMGB1 sofo ms $(13,14,31,50)$. Furthermore, it was reported recently that extracellular HMGB1 by itself may induce macrophage pyroptosis and caspase- 1 activation through RAGE-mediated endocytosis of HMGB1 (51). No information is yet available regarding HMGB1 isoform requirements for this positive feedback loop that may play an important functional role in the uncontrolled inflammation.

Administration of disulfide HMGB1 to normal animals produces systemic inflammatory responses with sickness behaviors including fever, anorexia, cognitive dysfunction, anemia, acute lung injury, endothelial cell activation, epithelial barrier dysfunction, arthritis and death (52). Therapeutic targeting of HMGB1 with neutralizing antibodies or other specific antagonists has been successful in numerous animal models of systemic inflammation (52-54).
The finding of our limited, retrospective study cannot provide definitive information about any critical step in the pathogenesis of life-thr _utening MAS, but rather represents a $f \cdot t$ a'tempt to explore dynamic changes of $\mathrm{F}$ GB1joforms in relationship to/clinical pry -ntation and given therapy. rulticer er studies are required to recruit re patient cohorts for prospe tive, randonized, controlled studies to $\mathrm{p}$ vide definitive conclusions on the 10. Hr_ -1 isoforms in MAS/HLH as biom. ars to predict outcome, treatment stratific tion and as possible target moleas for future therapy.

\section{ACKNOWLEDGMENTS}

This study was supported financially by grants through the regional agreement on medical training and clinical research (ALF) between Stockholm County Council and Karolinska Institute, the Swedish Association against Rheumatism, the Swedish Medical Research Council, Berth von Kantzow's Foundation, the Swedish Society of Medicine, Stiftelsen Allmänna Barnhuset and the Freemason Lodge Barnhuset in Stockholm.

Huan Yang wrote Ulf Andersson in 2015 to give permission for use of the personal communication.

\section{DISCLOSURES}

The authors declare that they have no competing interests as defined by Molecular Medicine, or other interests that might be perceived to influence the results and discussion reported in this paper.

\section{REFERENCES}

1. Ravelli A, Grom AA, Behrens EM, Cron RQ (2012) Macrophage activation syndrome as part of systemic juvenile idiopathic arthritis: diagnosis, genetics, pathophysiology and treatment. Genes Immun. 13:289-98.

2. Parodi A, et al. (2009) Macrophage activation syndrome in juvenile systemic lupus erythematosus: a multinational multicenter study of thirty-eight patients. Arthritis Rheum. 60:3388-99.

3. Simonini G, et al. (2010) Macrophage activation syndrome/hemophagocytic lymphohistiocytosis and Kawasaki disease. Ped. Blood Canc. 55:592.

4. Athreya BH. (2002) Is macrophage activation syndrome a new entity? Clin. Exp. Rheumatol. 20:121-3. 
5. Ramanan AV, Schneider R. (2003) Macrophage activation syndrome- - what's in a name! J. Rheumatol. 30:2513-6.

6. Stepp SE, et al. (1999) Perforin gene defects in familial hemophagocytic lymphohistiocytosis. Science. 286:1957-9.

7. Villanueva J, et al. (2005) Natural killer cell dysfunction is a distinguishing feature of systemic onset juvenile rheumatoid arthritis and macrophage activation syndrome. Arthritis Res. Ther. 7: R30-7.

8. Aouba A, De Bandt M, Aslangul E, Atkhen N, Patri B. (2003) Haemophagocytic syndrome in a rheumatoid arthritis patient treated with infliximab. Rheumatology (Oxford). 42:800-2.

9. Ramanan AV, Schneider R. (2003) Macrophage activation syndrome following initiation of etanercept in a child with systemic onset juvenile rheumatoid arthritis. J. Rheumatol. 30:401-3.

10. Scaffidi P, Misteli T, Bianchi ME. (2002) Release of chromatin protein HMGB1 by necrotic cells triggers inflammation. Nature. 418:191-5.

11. Harris HE, Raucci A. (2006) Alarmin(g) news about danger: workshop on innate danger signals and HMGB1. EMBO Rep. 7:774-8.

12. Bianchi ME, Manfredi A. (2004) Chromatin and cell death. Biochim. Biophys. Acta. 1677:181-6.

13. Lamkanfi M, et al. (2010) Inflammasome-dependent release of the alarmin HMGB1 in endotoxemia. J. Immunol. 185:4385-92.

14. Lu B, et al. (2012) Novel role of PKR in inflammasome activation and HMGB1 release. Nature. 488:670-4.

15. Takada H, et al. (1999) Oversecretion of IL-18 in haemophagocytic lymphohistiocytosis: a novel marker of disease activity. Br. J. Haematol. 106:182-9.

16. Venereau E, et al. (2012) Mutually exclusive redox forms of HMGB1 promote cell recruitment or proinflammatory cytokine release. J. Exp. $M_{1}$ 209:1519-28. 17. Yang $\mathrm{H}$, et al. (2012) Redox modif
teine residues regulates the cy 'ine a cys high mobility group box-1/ HM૦ Mol.ned. 18:250-9.

18. Antoine DJ, Harris $\mathrm{H}_{2}, \mathrm{~A}$ rsson $\mathrm{U}$, Iŕracey KJ, Bianchi ME. (2014) A system. nomenclature for the redox states of high mobslity group box (HMGB) pro 1 , M d. 20:135-7.

19. Yang $\mathrm{H}$, et al. (2o $\mathrm{A}$ cri cal cysteine is required for $\mathrm{HN}_{\mathrm{S}}$ inding Toll-like receptor 4 and act ion onnope cytokine release. Proc. Natl. A Sci. U.S. A. 107:11942-7.

20. Schiraldi al. (2012) HMGB1 promotes recruitment of inflammatory cells to damaged tissues by forming a complex with CXCL12 and signaling via CXCR4. J. Exp. Med. 209:551-63.

21. Bonaldi T, et al. (2003) Monocytic cells hyperacetylate chromatin protein HMGB1 to redirect it towards secretion. Embo. J. 22:5551-60.

22. Semino C, Angelini G, Poggi A, Rubartelli A. (2005) NK/iDC interaction results in IL-18 secretion by DCs at the synaptic cleft followed by NK cell activation and release of the DC maturation factor HMGB1. Blood. 106:609-16.

23. Semino C, et al. (2007) The maturation potential of NK cell clones toward autologous dendritic cells correlates with HMGB1 secretion. J. Leukoc. Biol. 81:92-9.

24. Li G, Liang X, Lotze MT. (2013) HMGB1: The central cytokine for all lymphoid cells. Front. Immunol. 4:68.

25. Lu B, et al. (2014) JAK/STAT1 signaling promotes HMGB1 hyperacetylation and nuclear translocation. Proc. Natl. Acad. Sci. U. S. A. 111:3068-73.

26. Ravelli A, et al. (2005) Preliminary diagnostic guidelines for macrophage activation syndrome complicating systemic juvenile idiopathic arthritis. J. Ped. 146:598-604.

27. Petty RE, et al. (1998) Revision of the proposed classification criteria for juvenile idiopathic arthritis: Durban, 1997. J. Rheumatol. 25:1 91-4.

28. Henter JI, et al. (2007) HLH-2004: Diagno, therapeutic guidelines for hemophagocytic ${ }_{1}$ phohistiocytosis. Ped. Blood Cance, 124-31.

29. Trottestam H, et al. (2011) Chemoin mu a apy for hemophagocytic lymphohistiocy osis: longterm results of the HLH-94 treatment fotocol. Blood. 118:4577-84.

30. Antoine DJ, et al 09 ) Hig nobility group box-1 protein and keratin ircul ing serum proteins informativ of acetamin en-induced necrosis and ap is vivo Toxicol. Sci.112:521-31.

31. Nys $\because \mathrm{m} \mathrm{S}, \quad(207)$ TLR activation regulates d age-associ. a molecular pattern isoforms rele during pyroptosis. EMBO J. 32:86-99.

Ge X, 2014) High-mobility group box-1 (HMGB1) Participates in the pathogenesis of alcoh lic liver disease (ALD). J. Biol. Chem. 280.22672-91.

Allen CE, Yu X, Kozinetz CA, McClain KL. (2008)

Highly elevated ferritin levels and the diagnosis of hemophagocytic lymphohistiocytosis. Ped. Blood Cancer. 50:1227-35.

34. Grom AA, Mellins ED. (2010) Macrophage activation syndrome: advances towards understanding pathogenesis. Curr. Opin. Rheumatol. 22:561-6.

35. Zoller EE, et al. (2011) Hemophagocytosis causes a consumptive anemia of inflammation. J. Exp. Med. 208:1203-14.

36. Shigemura T, et al. (2011) Monitoring serum IL-18 levels is useful for treatment of a patient with systemic juvenile idiopathic arthritis complicated by macrophage activation syndrome. Pediatr. Rheumatol Online J. 9:15.

37. Miettunen PM, Narendran A, Jayanthan A, Behrens EM, Cron RQ. (2011) Successful treatment of severe paediatric rheumatic disease-associated macrophage activation syndrome with interleukin-1 inhibition following conventional immunosuppressive therapy: case series with 12 patients. Rheumatology (Oxford). 50:417-9.

38. Schierbeck H, et al. (2013) HMGB1 levels are increased in patients with juvenile idiopathic arthritis, correlate with early onset of disease, and are independent of disease duration. J. Rheumatol. 40:1604-13.

39. Tamura K, et al. (2008) Increased serum monocyte chemoattractant protein-1, macrophage inflammatory protein-1b a, and interleukin-8 concentrations in hemo $\mathrm{c}$ is lymphohistiocytosis. Pea od C. ncer. 51:662-8.

40. Kazama H, et 亿l. (2008) Indu on of immunological tolerane poptotic cells requires caspasedepende pxida of ygh-mobility group box1 pro ein. Immunity. $\mathrm{s}: 21-32$.

41. Urb aviciute V, et al. (2009) Oxidation of the Glarn igh-m bility group box 1 protein $\mathrm{MGB}$, uaring apoptosis. Autoimmunity. 42. 7.

42. Antoi e DJ, Williams DP, Kipar A, Laverty H, Park BK. (2010) Diet restriction inhibits apoptosis nd HMGB1 oxidation and promotes inflammatory cell recruitment during acetaminophen hepatotoxicity. Mol. Med. 16:479-90.

43. Johnson TS, et al. (2014) Etoposide selectively ablates activated $\mathrm{T}$ cells to control the immunoregulatory disorder hemophagocytic lymphohistiocytosis. J. Immunol. 192:84-91.

44. Henter JI, et al. (2002) Treatment of hemophagocytic lymphohistiocytosis with HLH-94 immunochemotherapy and bone marrow transplantation. Blood. 100:2367-73.

45. Antoine DJ, et al. (2012) Molecular forms of HMGB1 and keratin-18 as mechanistic biomarkers for mode of cell death and prognosis during clinical acetaminophen hepatotoxicity. J. Hepatology. 56:1070-9.

46. Kim S, et al. (2013) Signaling of high mobility group box 1 (HMGB1) through toll-like receptor 4 in macrophages requires CD14. Mol. Med. 19:88-98.

47. Andersson U, et al. (2000) HMG-1 stimulates proinflammatory cytokine synthesis in human monocytes. J. Exp. Med. 192:565-70.

48. Sha Y, Zmijewski J, Xu Z, Abraham E. (2008) HMGB1 develops enhanced proinflammatory activity by binding to cytokines. J. Immunol. 180:2531-7.

49. Hreggvidsdottir HS, et al. (2009) The alarmin HMGB1 acts in synergy with endogenous and exogenous danger signals to promote inflammation. J. Leukoc. Biol. 88:655-62.

50. Yang H, Antoine DJ, Andersson U, Tracey KJ. (2013) The many faces of HMGB1: molecular structure-functional activity in inflammation, apoptosis, and chemotaxis. J. Leukoc. Biol. 93:865-73.

51. Xu J, et al. (2014) Macrophage endocytosis of high-mobility group box 1 triggers pyroptosis. Cell Death Differ. 21:1229-39.

52. Andersson U, Tracey KJ. (2011) HMGB1 is a therapeutic target for sterile inflammation and infection. Annu. Rev. Immunol. 29:139-62.

53. Yang H, Wang H, Czura CJ, Tracey KJ. (2005) The cytokine activity of HMGB1. J. Leukoc. Biol. 78:1-8.

54. Harris HE, Andersson U, Pisetsky DS. (2012) HMGB1: a multifunctional alarmin driving autoimmune and inflammatory disease. Nat. Rev. Rheumatology. 8:195-202. 